\title{
Human development dynamics: an agent based simulation of macro social systems and individual heterogeneous evolutionary games
}

\author{
Mark Abdollahian ${ }^{1}$, Zining Yang ${ }^{1 *}$, Travis Coan ${ }^{2}$ and Birol Yesilada ${ }^{3}$
}

* Correspondence:
zining.yang@cgu.edu
${ }^{1}$ School of Politics and Economics,
Claremont Graduate University, 170
E. Tenth St. Claremont, CA 91711,
USA
Full list of author information is
available at the end of the article

* Correspondence:

zining.yang@cgu.edu

Claremont Graduate University, 170

E. Tenth St. Claremont, CA 91711

Full list of author information is available at the end of the article

\begin{abstract}
Purpose: In the context of modernization and development, a complex adaptive systems framework can help address the coupling of macro social constraint and opportunity with individual agency. Combining system dynamics and agent based modeling, we formalize a simulation approach of the Human Development (HD) perspective to explore the interactive effects of economics, culture, society and politics across multiple human scales.
\end{abstract}

Methods: Based on a system of asymmetric, coupled nonlinear equations, we first capture the core qualitative logic of HD theory, empirically validated from World Values Survey (WS) data. Using a simple evolutionary game approach, second we fuse endogenously derived individual socio-economic attribute changes with Prisoner's Dilemma in an agent based model of the interactive political-cultural effects of heterogeneous, spatial intra-societal economic transactions. We then explore a new human development dynamics (HDD) model behavior via quasiglobal simulation methods to identify paths and pitfalls towards economic development, cultural plasticity, social and political change behavior.

Results: Our preliminary results suggest strong nonlinear path dependence and complexity in three areas: adaptive development processes, co-evolutionary societal transactions and near equilibrium development trajectories, with significant implications for anticipating and managing positive development outcomes. Strong local epistatic interactions characterized by adaptive co-evolution, shape higher order global conditions and ultimately societal outcomes.

Conclusions: Techno-social simulations such as this can provide scholars and policymakers alike insights into the nonlinear, complex adaptive effects of societal co-evolution. We believe complex adaptive or evolutionary systems approaches are necessary to understand both near and potentially catastrophic, far-from-equilibrium behavior and societal outcomes across all human scales of modernization.

Keywords: Economic development; Modernization; Cultural shift; Democratization; Game theory; Agent based model; Techno-social simulation; Co-evolution; Complex adaptive systems

\section{Springer}

C 2013 Abdollahian et al.; licensee Springer. This is an Open Access article distributed under the terms of the Creative Commons Attribution License (http://creativecommons.org/licenses/by/2.0), which permits unrestricted use, distribution, and reproduction in any medium, provided the original work is properly cited. 


\section{Background}

Since the turn of the 20th century, social scientists have identified dynamic linkages between economic development, cultural change, and political development with popularized arguments for and against modernization (Huntington 1996; Chua 2003; Sachs 2005). However, recent international events amplify the dynamism of social forces. Revolutions, international terrorism with economic development (Barnett 2004), instability via globalization (Rodrik 1998), modernization's "clash of civilizations" (Huntington 1996), the interlocks between politics and economics (Acemoglu and Robinson 2012) and the interdisciplinary Human Development (HD) perspective (Inglehart and Welzel 2005) are but some examples. Across all, positive feedback mechanisms shift seemingly stable system phenomena towards complexity and catastrophic, far-from-equilibria conditions (Thom 1977; Cambel 1993; Arthur 1994a, b;). Such events force our current scientific understanding to change perhaps radically, considering new possibilities and outcomes that integrate explanations across previously disparate fields.

Rooted in comparative political economy, the HD perspective is a qualitative, trans-disciplinary approach to understanding modernization and development through the lens of interdependent economic, cultural, social and political forces at multiple scales, from individuals to institutions and society as a whole. Here we extend previous work by Abdollahian et al.'s (2012) novel, quantitative systems dynamic representation of HD theory at the societal level towards integrated macro-micro scales in an agent based framework. As macroscopic structures emerging from microscopic events lead to entrainment and modification of both, co-evolutionary processes are created over time (Prigogine and Stengers 1984). Quek et al. (2009) also design an interactive macro-micro agent based framework, which they call a spatial Evolutionary Multi-Agent Social Network (EMAS), on the dynamics of civil violence. We posit a new, Human Development Dynamics (HDD) approach where agency matters: individual game interactions, strategy decisions and outcome histories determine an individual's experience. These decisions are constrained or incentivized by the changing macro economic, cultural, social and political environment via HD theory, conditioned on individual attributes at any particular time. Emergent behavior results from individuals' current feasible choice set, conditioned upon past behavior and macro outcomes. Conversely, progress on economic development, the formation of cultural mores, societal norms and democratic preferences emerge from individuals' behavior interactions.

In order to create a robust techno-social simulation (Vespignani 2009) platform, first we instantiate a system of asymmetric, coupled nonlinear difference equations that capture the core logic of HD macro-social theory. These are then empirically validated with five waves of data from the World Values Survey (2009). Similar to Quek et al. (2009), second we then fuse HD endogenous systems to agent attribute changes with a generalizable, non-cooperative Prisoner's Dilemma game following Axelrod (1987, 1997a, b), Nowak and Sigmund (1993, 1998, and Osterkamp (1997) to simulate intrasocietal, spatial economic transactions. Understanding the interactive political-cultural effects of macro-socio dynamics and individual agency in intra-societal transactions are key elements of a complex adaptive systems approach. Finally, we explore the model's behavioral dynamics via simulation methods to identify paths and pitfalls towards 
economic, social, cultural and political development as well as societal cooperation across different stages of development. We find strong epistatic interactions, where strategies are interdependent, and local social co-evolution (Kauffman 1993), help determine global-macro development outcomes in a particular society.

\section{HD dynamics background}

HD postulates a modernization process that is neither inevitable nor unidirectional. Following Maslow's (1954) hierarchy of needs, individual value orientations drive an individual's level of existential security and change in predictable ways given shifts in existential security. These value orientations are shaped by economic development and societal wealth. HD theory provides a framework in which economic development interacts with basic human needs and facilitates generalizable shifts in cultural predispositions and potentially revolutionary political behavior (Inglehart 1997; Inglehart and Baker 2000; Welzel et al. 2003; Inglehart and Welzel 2005). Robust empirics support that individual value orientation are represented by two primary dimensions - rationalsecular and self-expression value orientations (Inglehart 1997; Inglehart and Baker 2000; Welzel et al. 2003; Norris and Inglehart 2004; Inglehart and Welzel 2005). Both are strongly influenced by economic progress and are more prevalent at different stages of development. HD theory naturally lends itself to a complex adaptive systems approach given the theoretical entrainment of macroscopic structures and microscopic individual agency, with individual value orientations shaped by and impacting macro societal outcomes.

While not a strict, formal economic growth model, HD theory leverages off growth theory. Neoclassical growth models (Solow 1956; Swan 1956; Barro 1991) specify three key economic drivers as the engine of growth: increasing capital relative to labor, higher rate of returns for underdeveloped societies and finally diminishing returns to capital. Together these factors propel middle income societies on high growth paths towards convergence and steady state equilibrium - measured as sigma convergence which is the reduction in income dispersion levels across economies and beta convergence when developing societies growth rates slow down as they become developed (Mankiw et al. 1992; Sala-i-Martin 1996). Such approaches specify detailed and interactive vectors of economic determinants, with country and time specific effects separate and in a more auxiliary manner (Caselli et al. 1996). Dissatisfied with a theoretically exogenous and ad hoc role of technology decoupled from economic factors, endogenous growth scholars (Romer 1986; Lucas 1988) explicitly link technology and human capital's effects to productivity. One major finding is that increasing rates of return to human capital do not produce steady state outcomes nor convergence. This allows highly developed societies to continue growing.

Similar to the endogenous growth literature, HD theory expands the breadth of development processes to include cultural, social and political effects to account for modernization across all scales. First, rational-secular values correspond to individuals' growing emphasis on technical, mechanical, rational, and bureaucratic views of the world. During economic industrialization phases, cultural dispositions tend to progress from an emphasis on traditional pre-industrial values-often measured in terms of religious ceremony attendance and the importance of religion in an individual's life-to 
secular world views, transferring authority from traditional religious figures to technological progress and bureaucratic political life.

The second dimension, self-expression, corresponds to the post-industrial phase of economic development where economic progress and an advanced welfare system provide many individuals in with an overwhelming sense of existential security (Bell 1973). As economic activity changes from primary, to secondary and tertiary sectors, individuals no longer work on farms, but in factories, while some pursue careers in the service industry. The reduction in human constraint-via increased productivity and wealth, advances in education, and service related economic activities-brings about a new sense of autonomy. Because the primary focus is no longer on survival, individuals are free to emphasize a general need for self-expression, question authority, and demand political participation. Rising self-expression values lead to the emergence of effective political institutions (Welzel et al. 2003). Mass tendencies toward self-expression facilitate a political climate conducive to potentially elite-challenging activity and a civic culture consistent with genuine democratic governance.

Self-expression values promote liberal political institutions through two mechanisms. First, to the extent that there is incongruence between cultural demand for, and political supply of liberal institutions, individuals are more or less prone to elite-challenging activity (Gurr 1970; Eckstein and Gurr 1975). Second, self-expression values support the social acceptance of basic democratic norms such as trust and political participation. The end result is a gradual transition toward democratization in autocratic nations and more effective political representation in democratic nations (Inglehart and Welzel 2005). Lastly, based on work on liberal institutions and economic development (Diamond 1992; Boix 2003; Feng 2003; Acemoglu and Robinson 2012) HD theory expects positive feedbacks between democratic institutions and economic progress. Declining economic conditions reintroduce the primacy of basic economic needs, fueling the structural conditions for more traditional value orientations, reducing society's ability to seek self-actualization, and increasing the likelihood of political change. Disequilibrium between culturally defined political expectations and political rights or civil liberty realities promotes elite challenging activity and provides motivation for revolutionary change. Equations 1 through 4 below transform HD's qualitative theoretical specification into a nonlinear, first order interdependent system (Abdollahian et al. 2012).

$$
\begin{aligned}
& \frac{d Y}{d t}=\lambda_{1}(1-Y)+\lambda_{2} D \\
& \frac{d R S}{d t}=\left[\alpha_{1} Y-\alpha_{2}(R S-Y)\right] R S(1-R S) \\
& \frac{d S E}{d t}=\beta_{1} Y S E(1-S E) \\
& \frac{d D}{d t}=\left[\gamma_{1} S E(S E-D)+\gamma_{2} D\right](1-D)
\end{aligned}
$$

$Y$ economic progress 
$R S$ rational-secular values

$S E$ self-expression values

$D$ effective democracy

$\alpha_{1}$ maps the interactive effects of $\mathrm{Y}$ and RS onto growth in level of RS

$\alpha_{2}$ regulates the dynamic effects of economic incongruence

$\beta_{1}$ maps the interactive effects of $\mathrm{Y}$ and SE onto growth in the level of SE

$\gamma_{1}$ regulates the dynamic effects of political incongruence

$\gamma_{2}$ maps past governance structure onto the growth in effective democracy

$\lambda_{1}$ parameter for economic progress

$\lambda_{2}$ parameter of the democratic impact on economic development

Figure 1 above identifies four macro HD system phase portraits (Abdollahian et al. 2012) under equilibrium conditions. The HD perspective suggests a staged process in which rising level of existential security via economic development leads to an increased emphasis on rational-secular and self-expression values. Specifically, individuals tend to emphasize rational-secular values during the industrialization phase of development and self-expression values during the post-industrial phase. Second, the rise in self-expression values strengthens democratic norms and promotes effective democracy, implying a positive relationship between self-expression and liberal political institutions. However, these effects are neither linear nor monotonic as we see strong reversion towards autocratic institutional preferences in low self-expressive, survival

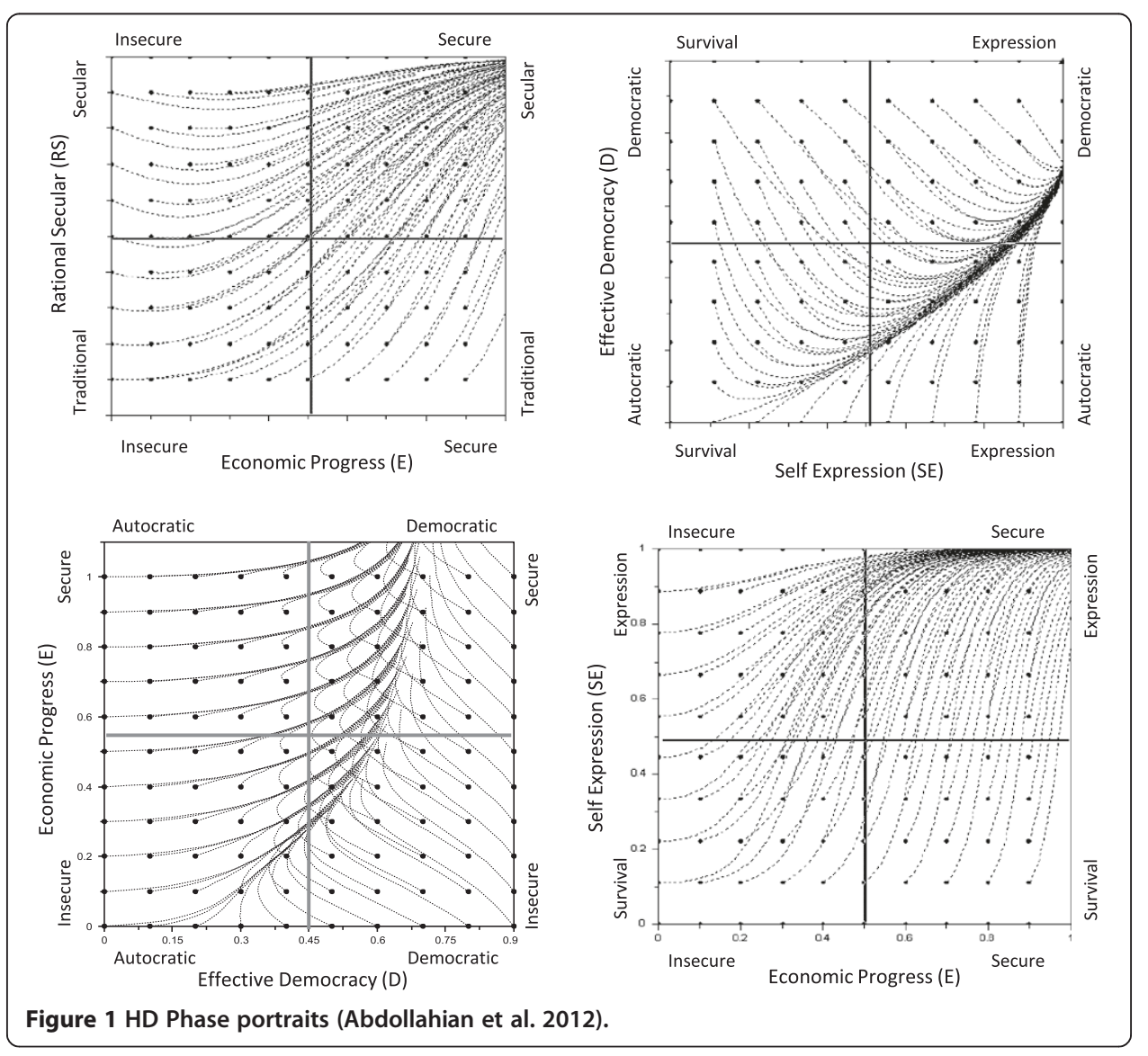


dominated societies. Key to driving effective democracy are sustainable levels of expressive values. Democratic norms and institutions that outpace economic progress are inherently unstable with a persistent, turbulent reversion processes. This can occur even at high levels of democratic norms and existential security. This suggests societies experiencing democratization can frequently expect punctuated reversals and revolutions towards more autocratic institutions until more sustainable economic growth and democratic institutions re-emerge.

\section{Methods}

While innovative and the first to formalize a systems approach for HD theory, a limitation of Abdollahian et al's (2012) work lacks, coupling and interdependence across human scales, from individuals to institutions and finally the societal outcomes they generate. Our HDD model uniquely combines the interactive effects and feedbacks between individual human agency as well as the macro environmental constraints and opportunities that change over time for any given society. Decisions by individuals are affected by other individuals, social context, and system states. These decisions have variegated first and second order effects, given any particular system state or individual attributes. Such an approach attempts to increase both theoretical and empirical verisimilitude for some key elements of complexity processes - emergence, connectivity, interdependence and feedback (Miller and Page 2007) - found throughout several disciplines across all scales of modernization and human development.

Implemented in NetLogo (Wilensky 1999), Figure 2 depicts the high level process and multi-module architecture. We maintain individual agent attribute relationships and postulated changes of RS, SE, $D$ and $Y$ following HD theory. These endogenously derived, individual agent attributes $\left(R S^{i}, S E^{i}, D^{i}\right.$ and $\left.Y^{i}\right)$ impact how economic

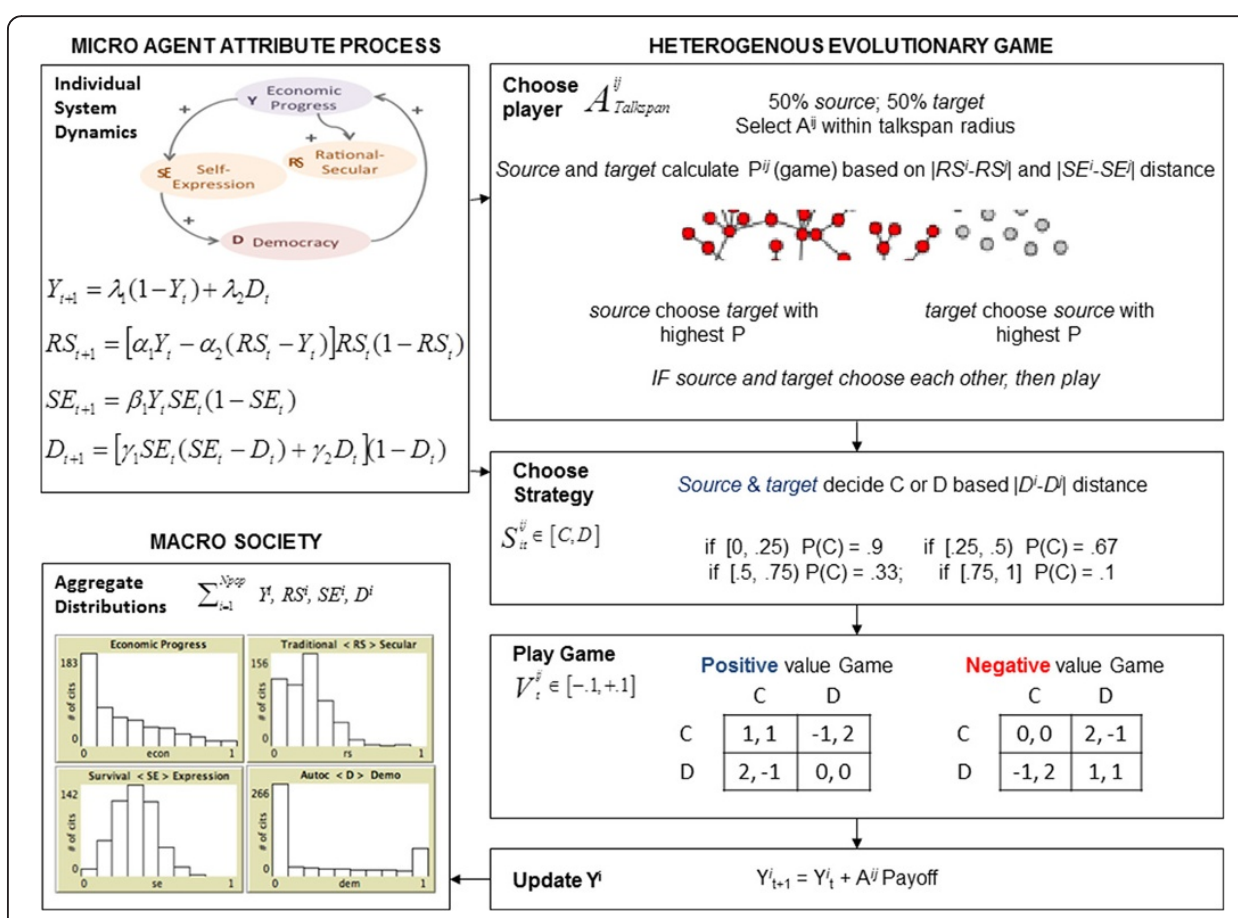

Figure 2 HDD architecture. 
transaction games occur, either increasing or decreasing individual wealth and at increasing scales, ultimately societal productivity (Binmore 1998). Thus we create the tessellated pattern of heterogeneously mixed populations that cooperate and compete in a changing political economy backdrop to model social co-evolutionary processes

Capturing individual agent endogenous processes, we first transform Abdollahian et al.'s system of equations from differential to discrete equations for NetLogo tractability and use their empirically validated parameter values as a good first approximation. Given individual citizen attributes and HD processes at each iteration, we sum up each agent attributes across $Y, R S, S E$ and $D$ to find resulting societal distributions for each variable, yet are mindful of ecological correlation. This allows us to explore the interactive effects of income inequality, cultural schisms, social complexity or highly polarized political dialogues in any given society as the emergence of individual efforts and patterns of interactions.

Evolutionary game theory provides insights to understanding individual, repeated societal transactions in heterogeneous populations (Fudenberg and Maskin 1986; Sigmund 1993; Maynard-Smith and Szathmary 1995). Social co-evolutionary systems allow each individual to either influence or be influenced by all other individuals as well as macro society (Snijders et al. 2007; Zheleva et al. 2009), perhaps eventually becoming coupled and quasi-path interdependent. In our case, we do not have well mixed populations, but explicit spatial contact networks given population density, technology diffusion and agent attributes. Thus we explicitly recognize that the differential impact of heterogeneous, spatial structures matters (Santos et al. 2006). This captures various individual preferences and their socioeconomic attributes. Accordingly, we instantiate a non-cooperative, socio-economic Prisoner's Dilemma (PD) transaction game given agent $i$ 's attribute vector $\left(\mathrm{A}^{i}\right)$ of social, cultural, political and economic preference $\left(R S^{i}, S E^{i}, D^{i}\right.$ and $\left.Y^{i}\right)$ similarity to agent $j\left(\mathrm{~A}^{j}\right)$ for any $\mathrm{A}^{i j}$ pairs. The motivation behind this is that individuals are more likely to interact, engage and conduct transactions with other agents of similar religious, secular and expressive norms (Schelling 1971) and produce different co-evolutionary behavior via frequency and rate dynamics (McKelvey 1999).

At each iteration $t$, we first randomly choose $50 \%$ of spatially proximal agents as sources who can select a partner. The remaining targets are chosen by other agents based on symmetric preference rankings and asymmetric neighborhood proximity distributions. Here we look at communications reach, social connectivity and technology diffusion as constraining the potential set of $\mathrm{A}^{i j}$ game pairs, called talk span. Low values constrain games to local neighborhoods among spatially proximate agents, while higher values expand potential $\mathrm{A}^{i j}$ pairs globally, modeling socially compressed space.

Social Judgment Theory (Darity 2008; Griffin 2009) describes how the positions of two agents can be conceived along a Downsian continuum while distance between these positions affects the likelihood of one accepting the other's position. Agent $i$ evaluates the likelihood of conducting a simple socio-economic transaction with agent $j$ based on similarity of socio-cultural preferences $\left|R S^{i}-R S^{j}\right|$ and $\left|S E^{i}-S E^{j}\right|$ within a given local neighborhood. This captures communications and technology diffusion for frequency and social tie formation (McPherson et al. 2001). This also reflects recent work on the importance of both dynamic strategies and updating rules based on agent attributes affecting co-evolution (Kauffman 1993; Moyano and Sanchez 2008). Source 
agents evaluate $R S$ and $S E$ distances separately between themselves and all target agents within a given neighborhood radius. Shorter preference distances increase the probability that $A^{i j}$ will enter into a socio-economic transaction and play the PD game. After each source agent calculates its probability of playing a game with all possible target agents, it chooses the target with the highest probability as its partner. Target agents also repeat the same process symmetrically. We then choose the $\mathrm{A}^{i j}$ pairing with the highest probability derived from its preference-proximity function at a particular iteration.

After agents decide to play at each $t$, each select strategies probabilistically based on similarity of political preferences as expressed by $\left|D^{i}-D^{j}\right|$. Siero and Doosje (1993) among others show that messages close to a receiver's position has little effect, while those far from a receiver's position is likely to be rejected, capturing the notions of bipartisanship or conversely hyper-partisanship. So when $D$ distance is short, there is a higher probability of cooperating while larger distances results in a higher probability of defecting. Relative payoffs for each agent are based on simple PD, non-cooperative game theory (Nowak and Sigmund 1993; Fehr and Fischbacher 2004; Dixit et al. 2009) where $\mathrm{T}>\mathrm{R}>\mathrm{P}>\mathrm{S}$, with $\mathrm{T}=2, \mathrm{R}=1, \mathrm{P}=0$ and $\mathrm{S}=-1$. When both agents cooperate, each agent receives a payoff of $\mathrm{T}$. When one plays cooperate but the other plays defect, the cooperating agent loses with payoff $\mathrm{S}$ while the defecting agent gains with payoff $\mathrm{T}$. When both play defect, neither gain from the transaction, and each agent receives a payoff of $\mathrm{P}$.

Following Nowak and Sigmund (1993), we randomly assign game transaction values. However we do not asymmetrically constrain such values, instead allowing any particular game transaction value between pairs, $\mathrm{V}^{i j}$, to lie in between $[-.1, .1]$, randomly distributed. This instantiation allows for different potential deal sizes, costs, benefits, or synergies for any agent to gain or lose. We specifically model socio-economic transaction games as producing either positive or negative values as we want to capture behavioral outcomes from games with both upside gains or downside losses. Subsequently, $\mathrm{A}^{i j}$ games' $V^{i j}$ outcomes condition agent $E_{t+1}^{i}$ values, modeling realized costs or benefits from any particular interaction. The updated $E_{t+1}^{i}=E_{t}^{i}+A^{i j}$ game payoff for each agent then gets added to the individual's attributes for the next iteration. We then repeat individual endogenous processing, aggregated up to society as a whole and repeat the game processes for $t+n$ iterations, where $n$ is the last iteration.

In our HDD framework, $A_{i}$ strategies are adaptive, which affect $A_{i j}$ pairs locally within a proximate radius as first order effects. Other agents, within the system but outside the talkspan radius, are impacted through cascading higher orders. In HDD, we explicitly model epistatic interactions (Kauffman 1993) to capture co-evolutionary behavior in a simple, yet elegant manner. Although easily done, we specifically do not model mathematically complex, individual agent memory or learning from $V^{i j}$ outcomes as many others do (Axelrod 1987; Sigmund and Nowak 1999). However, memory and history still matter. The sum of all prior individual behavioral histories, evolutionary through iterations, does contribute to each individual and societal current states. As an initial effort at a scale integrated framework, this frees us to focus on the coupling of structure and agency first, before enriching subcomponent process detail. Thus agents simultaneously co-evolve as strategy pair outcomes CC, DC/CD or DD at $t$ to increase $Y^{i}$ at $t+1$, thus driving both positive and negative $R S, S E$ and $D$ 
feedback process through $t+n$ iterations. These shape $A^{i}$ attributes which spur adaptation to a changing environment, summing $Y^{i}, R S^{i}, S E^{i}$ and $D^{i}$ vector values. Feedback into subsequent $A^{i j}$ game selection networks and strategy choice yields a complex adaptive system representation across multiple scales. The accompanying pseudo code is shown below.

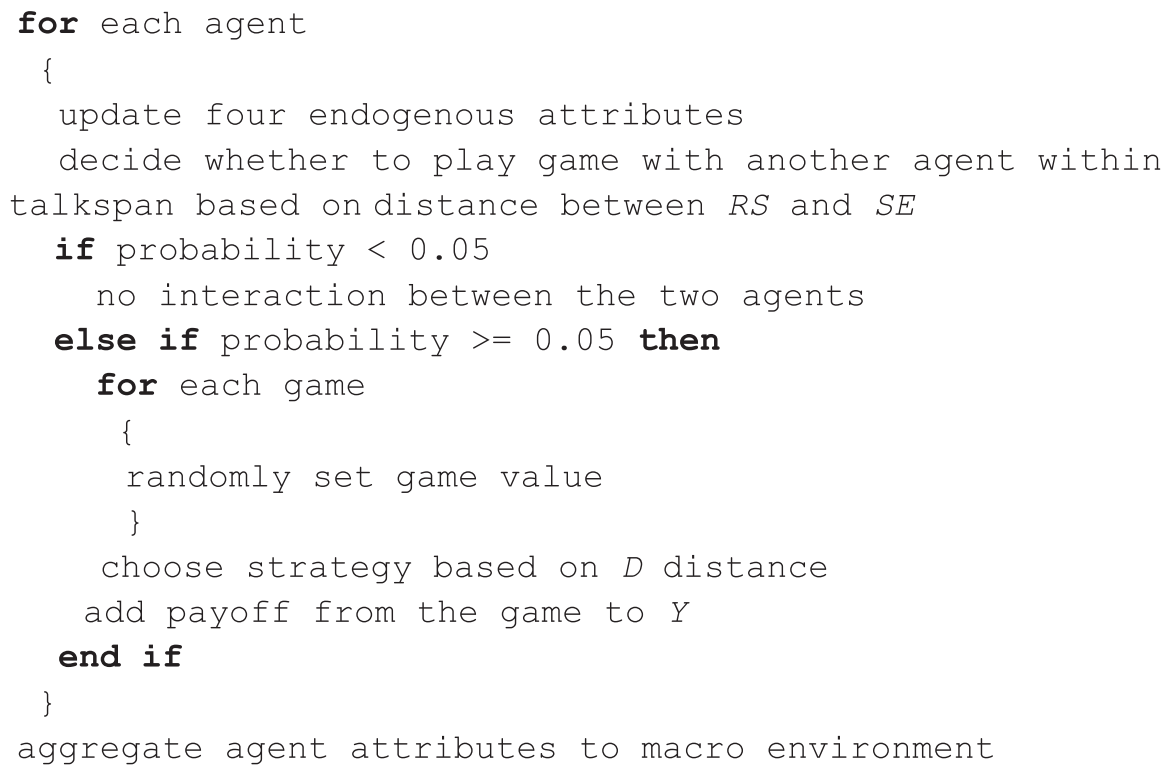

\section{Results}

We use Abdollahian et al's parameter estimates from their genetic algorithm non-linear least squares (GANLS) estimation procedure on WVS data to populate system coefficients to increase model validity. The data spans from 1981 to 2007 from five waves for over 45 countries. The GANLS three step approach (Coan 2011) first employs a genetic algorithm to search global parameter space, a simplex algorithm to search local parameters space, and finally a bootstrapping procedure to derive the uncertainty associated with the parameter estimates. Given empirical $\beta$ coefficient estimates for the HD system, we then conduct a quasi-global sensitivity analysis on the entire HDD agent based model input and initial condition parameters for 700 time steps using pooled OLS regression. Here we approximate one time step as one month given past data calibration (Abdollahian et al. 2012) for a simulated time span of almost 60 years. We performed over 40,000 runs given various input parameter combinations for a low resolution, initial sensitivity approximation.

Figure 3 depicts our HDD interface and a single sample run. The interface shows physical output space-heterogeneously mixed agents distributed spatially, where agent size indicates individual economic wealth $Y$, agent color represents democratic preference $D$, ranging from fully autocratic in red to fully democratic in blue. $A^{i j}$ game transactions $V^{i j}$ are shown as links at each $t$. For all agents' $Y, R S, S E, D$ attributes, we setup the following initial conditions: societal mean $(\bar{Y}, R \bar{S}, S \bar{E}, \bar{D})$ and standard deviation, population density and techno-social connectivity via talkspan. Although not detailed 

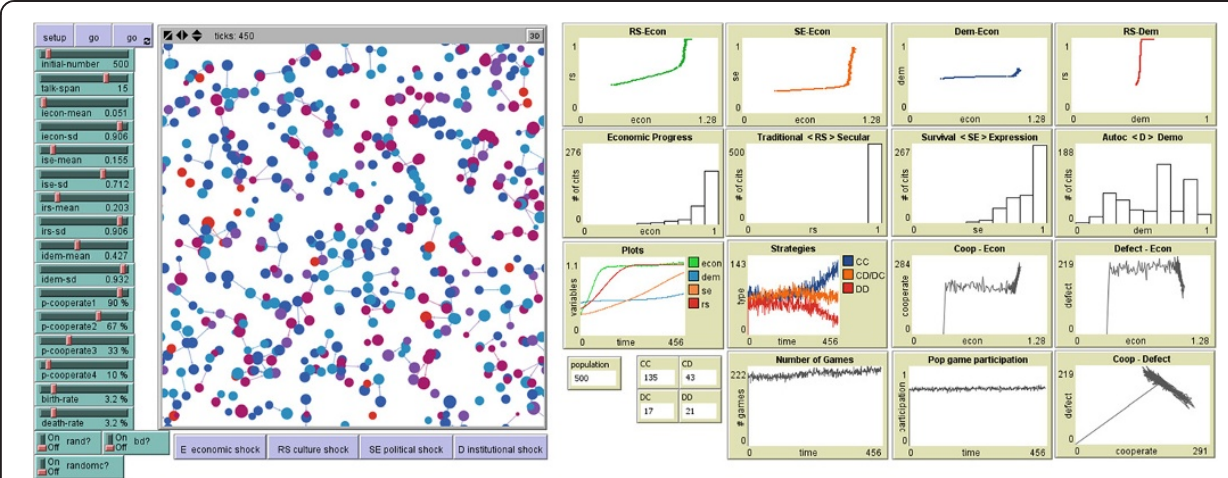

Figure 3 Sample HDD run.

herein, we also parameterize different agent birth and death rates and probability of game interactions in addition to strategies that can be randomized individually or in combinations to isolate dynamics in any specific subcomponent module.

Monitors and plots include time series and dynamic phase portraits for the societal average of $Y, R S, S E$ and $D$ trajectories and dynamic histograms across all attributes. This allows us to trace the development of wealth, income inequality, cultural dispositions, and political polarization. We also track the number of transactions games, the percentage of population interacting, and the number of different cooperative, mixed, or non-cooperative strategy pairs under different macro environmental conditions.

Before turning to our sensitivity results, we detail our notional run. Here a lesser developed society, with a mean low income level but high degree of inequality, escapes the poverty trap through high growth and increasingly moderating democratic institutions. Individual productivity and wealth, driven by successful CC and DC/CD strategy outcomes of individual transactions, help accelerate the emergence of modern secular $R S$ and expressive $S E$ norms and values, while democratic $D$ preferences solidify, but are not engrained throughout all of society. Figure 4 depicts spatial details from the above simulation at $t=0,150,300$ and 450 snapshots. Initially, we find our low developed society with high income inequality and polarization along autocratic and democratic preferences. By $t=150$, agents quickly increase in individual wealth as several economic transaction games are played between individuals of similar $R$ and $S$ preferences while the strategy history shows not one dominant strategy emerges as society goes through a high growth phase of development. By $t=300$, we see continued heterogeneously mixed populations in terms of income and democratic preferences, while by $t=400$ we see increased convergence toward democratic preferences. Although just one particular simulation, what is critical is that co-evolutionary behavior results in path dependence of economic and cultural change as well as being a key determinant for development outcomes. Moreover, changes towards democratic values leads to increasing cooperative strategy pairs over time.

\section{Sensitivity analysis}

In order to make more generalizable model inferences, Table 1 details the interactive parameter effects on economic prosperity $Y$, as well as strategy choice pairs $\mathrm{CC}, \mathrm{CD} / \mathrm{DC}$ and 


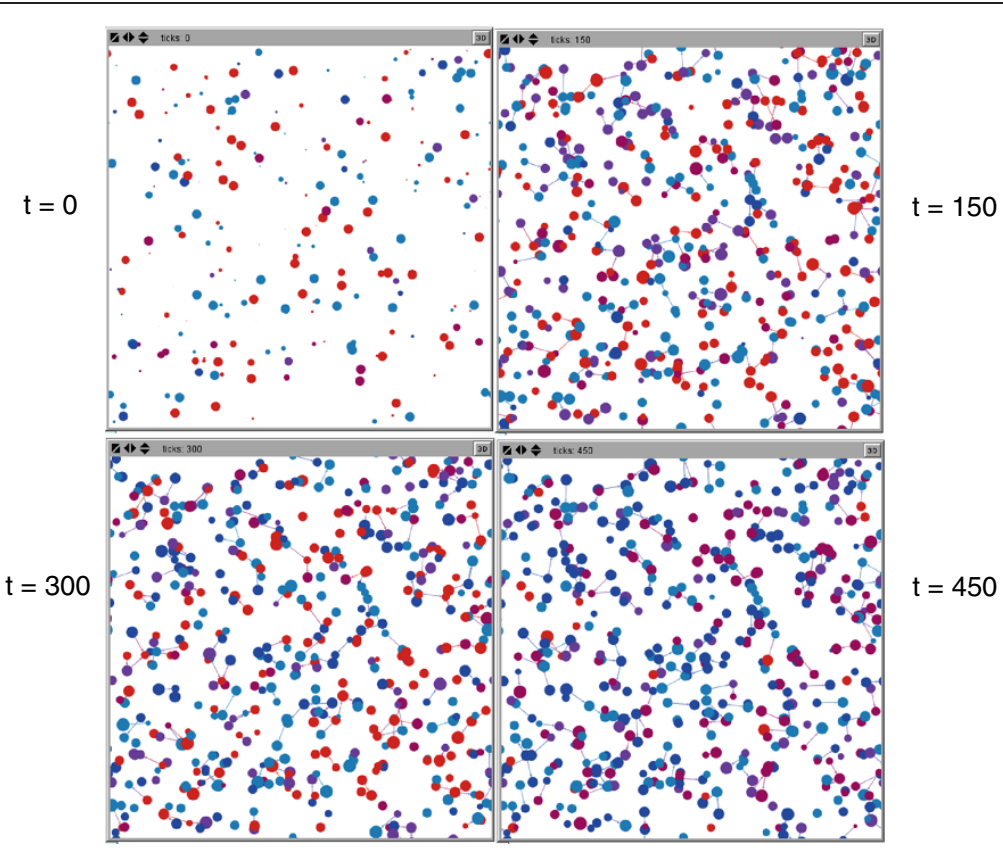

Figure 4 Spatial outcomes at $t=0,150,300$ and 450 .

Table 1 Impact on economic development and strategy pairs

\begin{tabular}{|c|c|c|c|c|}
\hline Model & Economic & $\mathrm{CC}$ & $C D$ & DD \\
\hline \multirow[t]{2}{*}{ Economic } & & $0.3846^{*}$ & $0.2467^{*}$ & $0.1032^{*}$ \\
\hline & & (0.0019) & $(0.0015)$ & $(0.0015)$ \\
\hline \multirow[t]{2}{*}{ Rational secular } & $0.3298^{*}$ & $-0.2756^{*}$ & $0.0598^{*}$ & $0.0643^{*}$ \\
\hline & $(0.0022)$ & $(0.0022)$ & $(0.0020)$ & $(0.0021)$ \\
\hline \multirow[t]{2}{*}{ Self expressive } & $0.1488^{*}$ & $0.2139^{*}$ & $-0.0120^{*}$ & $-0.1571^{*}$ \\
\hline & $(0.0015)$ & $(0.0016)$ & $(0.0014)$ & $(0.0014)$ \\
\hline \multirow[t]{2}{*}{ Democratic } & $0.1161^{*}$ & $0.0623^{*}$ & $-0.0790^{*}$ & $-0.0337^{*}$ \\
\hline & $(0.0016)$ & $(0.0018)$ & $(0.0015)$ & $(0.0015)$ \\
\hline \multirow[t]{2}{*}{ Cooperate } & $0.1606^{*}$ & & & \\
\hline & $(0.0006)$ & & & \\
\hline \multirow[t]{2}{*}{ Defect } & $0.1088^{*}$ & & & \\
\hline & $(0.0011)$ & & & \\
\hline \multirow[t]{2}{*}{ Talk-span } & $0.1615^{*}$ & $0.5484^{*}$ & $0.3024^{*}$ & $0.1306^{*}$ \\
\hline & $(0.0005)$ & $(0.0010)$ & $(0.0007)$ & $(0.0007)$ \\
\hline \multirow[t]{2}{*}{ Time } & $0.0558^{*}$ & $0.1742^{*}$ & $-0.2414^{*}$ & $-0.1958^{*}$ \\
\hline & (0.0009) & $(0.0013)$ & $(0.0009)$ & $(0.0007)$ \\
\hline \multirow[t]{2}{*}{ Constant } & $0.1972^{*}$ & $-0.2481^{*}$ & 0.0028 & $0.1353^{*}$ \\
\hline & $(0.0019)$ & $(0.0017)$ & $(0.0015)$ & $(0.0016)$ \\
\hline \# of obs & 634459 & 634459 & 634459 & 634459 \\
\hline Prob $>F$ & 0.0000 & 0.0000 & 0.0000 & 0.0000 \\
\hline R-squared & 0.6441 & 0.6468 & 0.5171 & 0.3442 \\
\hline Root MSE & 0.1285 & 0.2081 & 0.1500 & 0.1291 \\
\hline
\end{tabular}

Numbers in parentheses are corresponding robust standard errors.

* Significance at $1 \%$ level. 
DD. Our quasi-global sensitivity analysis generated over 27 million observations that we randomly down sample to approximately 634,000 for pooled OLS tractability. As all variables are relatively scaled, we can interpret magnitude and substantive effects across OLS $\beta$ coefficients.

Our first model on economic development $Y$ confirms HD theory that positive values of mean societal $R S, S E$ and $D$ values do significantly speed the pace of economic development, with $R S$ providing the most substantive effect $(\beta=.3298)$. Looking at the impact of evolutionary games, we see the number agents both choosing cooperation has a stronger positive impact $(\beta=.1606)$ than defection $(\beta=.1088)$ in increasing societal economic value. This suggests that cooperation does pay higher social dividends on average. Talkspan spatial proximity is positive and significant $(\beta=.1615)$, confirming priors that increasing technology and compressing potential social space also speed development processes. Time is also slightly positive $(\beta=.0558)$, indicating that economic prosperity is quasi self-reinforcing and path dependent. Model fit $\left(R^{2}=.6441\right)$ is acceptable given the highly complex and non-linear dynamics and pooled nature of sensitivity analysis data.

Next we explore the potential for co-evolutionary behavior across micro and macroscopic scales. Turning to HDD parameter effects on heterogeneously mixed evolutionary games, we first focus on the conditions associated with CC strategy pairs - number of agent interactions where both $i$ and $j$ chose to cooperate. Talkspan is the most substantial $(\beta=.5484)$, indicating increasing individual agents' ability to reach other likeminded agents spurs cooperation dramatically based on first order local interactions. Not surprisingly, economic progress $Y(\beta=.3846)$ and self-expression $S E(\beta=.2139)$ are also influential on increasing cooperative societal transactions showing coupled and cascading emergence at increased scale. Unexpectedly, secular values $R S(\beta=-.2756)$ significantly decrease societal cooperation but still exhibit strong co-evolution, perhaps supplanting contract enforcement in traditional societies. Time is significantly positive ( $\beta=.1742)$, showing how cooperation tends to evolve over time with path dependence. This is especially interesting in light of $R S$ 's positive impact on growth. This suggests that non-Pareto, asymmetric payoffs might be a necessary condition for development, regardless of path dependency. Model fit $\left(R^{2}=.6468\right)$ is acceptable.

Looking at the number of agent interactions where either $i$ or $j$ cooperates when the other defects (CD or DC), we find strong co-evolution but with the different effects. While talkspan still dominates locally $(\beta=.3024)$ and the economic environment ( $\beta=.2467)$ influences the nature of strategic interactions, there is less relative impact in driving asymmetric agent strategies. Consistently, increasing secular values SE $(\beta=.0598)$ slightly contributes to asymmetric strategic behavior while increasing democratic norms $D(\beta=-.0790)$ and expressive $R S$ behavior $(\beta=-.0120)$ curtails such unilateral advantages. More importantly, time $(\beta=-.2414)$ is highly negatively related to asymmetric payoffs. Under asymmetry, agents cannot sustain such transaction outcomes as economic gains motivate preferences to become more closely aligned. Model fit $\left(R^{2}=.5171\right)$ is also acceptable.

Finally, focusing on the number of agent interactions where both $i$ and $j$ chose defection (DD), we find self expressive SE behavior $(\beta=-.1571)$ and time $(\beta=-.1958)$ have the largest impact in dampening asymmetric payoffs in societal transactions. Democratic institutions $D(\beta=-.0790)$ also still deter such behavior. Talk span 
( $\beta=.1306)$ and economic progress $Y(\beta=.1032)$ as expected increase co-evolving DD strategy pairs but surprisingly at much lower levels than either in $\mathrm{CD} / \mathrm{DC}$ or $\mathrm{CC}$ transactions. Expanding the feasible spatial set and individual's wealth does increase the temptation to defect for both agents. It also raises the immediacy of first order effects. Model fit $\left(R^{2}=.3442\right)$ is relatively poor on DD strategy pairs compared to our other results, suggesting more complex processes need further exploration to increase result confidence.

\section{Discussion}

Figure 5 depicts a panel of average run trajectories across sampled parameter space. Here we focus on the interactive effects of low, medium and high mean self expressive $(\overline{S E}=.05, .5, .95)$ behavior plots across societies with differing low, medium and high mean democratic norms $(\bar{D}=.05, .5, .95)$ given various levels of economic development and mutually cooperative $(\mathrm{CC})$ behavior. Each line represents the average iteration value between $t=0$ to 700 of our 40,000 runs. Low, middle and high mean developed $(\bar{Y}=.05, .5, .95)$ societies trajectories are in blue, orange and green respectively. Regardless of initial macro social conditions and consistent with neoclassical economic growth theories, we see both sigma income levels and beta growth rates conditional convergence with similar trajectory slopes and phase shifts. We also find

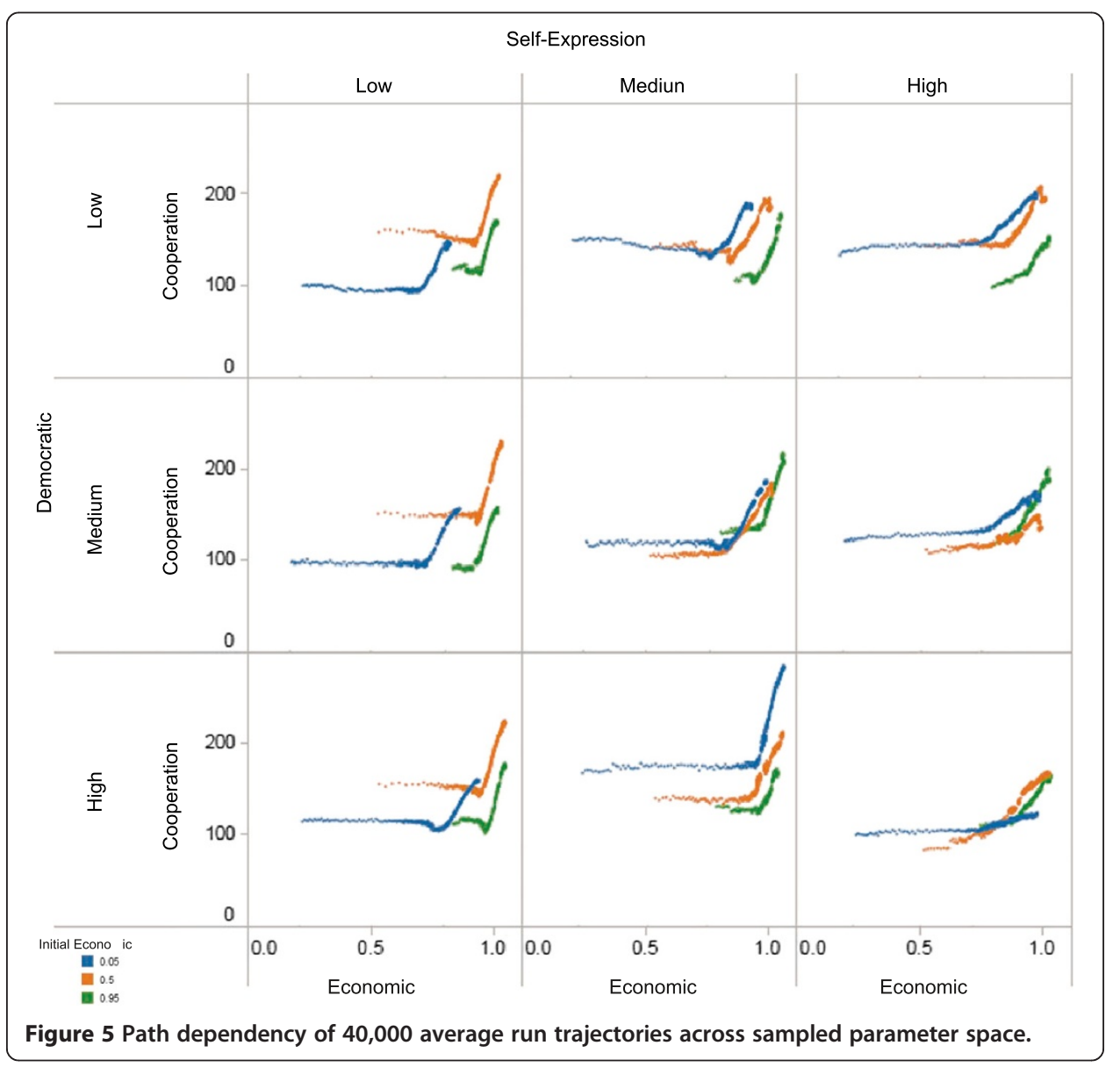


cooperation does take time to emerge across all plots, indicating increasing entrainment of social co-evolution. However, focusing on highly developed societies (in green) across all plots, we see on average, intra-societal cooperative transactions emerge faster than for low income societies (in blue). This does not occur necessarily at higher development levels as our previous results suggest income growth, not levels, is a key driver of CC strategy pairs. Looking across initial democratic societal conditions, development outcomes and cooperative norms are most varied in low $S E$ societies and tend to homogenize as individual and societal expressive culture increases. More interestingly, democratic institutions actually provide the largest impetus, fostering more cooperation and increasing growth in low and middle-income societies. These effects are both time and path dependent as they diminish in highly expressive, democratic societies where economic factors once again become the key determinant.

\section{Conclusions}

Consistent with qualitative HD theory and empirical reality, our HDD model finds complexity and nonlinear path dependence in three areas: adaptive development processes, social co-evolutionary transactions and near equilibrium development trajectories. From a complex adaptive system perspective on HD theoretical processes, economic progress is a necessary condition for successful secularization and expressive political behavior, which are antecedents for lasting democratic institutions. While modernization is not inevitable, our results support empirical observations for a staged process where increasing existential security via economic development leads to increased emphasis on rational-secular and self-expressive values that results in societal development. Here we find that rational-secular norms strongly impact economic growth and speed up the pace of development more than self-expressive societal values alone. Beyond supporting HD theory, agents do adapt interactively with their environments as mutual cooperation does result in higher societal wealth than defection alone and is self reinforcing over time.

Exploring the impact of societal conditions on transactions, in CC strategy pairs, compressing techno-social space by increased spatial proximity promotes co-evolutionary entrainment and mutual cooperation. Surprisingly, high secular values actually dampen $\mathrm{CC}$ outcomes suggesting the necessary role of asymmetric, Nash dominant strategies as one potential engine of high growth during industrialization phases. For $\mathrm{CD} / \mathrm{DC}$ strategy pairs, we find consistently similar factors but with converse impacts. More interestingly, increasing macroscopic secular values over time has a dampening effect on individual non-Pareto outcomes. Higher macroscopic societal wealth and expanded social connectivity also dampen DD outcomes initially.

Turning to the path dependence of development trajectories, our results are consistent with neo-classical growth income convergence and the evolution of cooperation (Axelrod 1987, 1997a). We find convergence despite various initial income levels, inequality, social and cultural conditions on both sigma levels and beta growth rates while cooperation emerges over time. Social co-evolution of Pareto outcomes as seen by CC strategy pairs, tends to materialize faster in low income countries then dissipate in high growth, middle income societies only to return towards more predominately Pareto transaction outcomes in high income societies. One possible explanation is that 
asymmetric payoffs and non-cooperative behavior might be a necessary condition for high growth, emerging market economies. Epistatic Pareto outcomes and resulting wealth creation are most varied in low self-expressive societies, consistent with characterizations of a poverty trap (Sachs 2005). Democratic norms and institutions are a key driver, providing the largest impact in fostering cooperation and speeding economic growth in low and middle income societies. However, we find diminishing returns to democratization's benefits in advanced, highly expressive and already democratic societies, when economics once again takes primacy.

As economic activity pushes into the quaternary phase of development, we can only begin to ponder potential implications. Transitioning knowledge based services, and the multiplier impact that technology has on culture, politics and human development, only accelerates discontinuous change. Such forces are likely to produce new positive feedbacks across previously disparate and isolated domains, amplifying the coupling of technology, culture and society, to induce far-from-equilibrium conditions that will surprise us the most. Punctuated equilibria changes are the norm, not the exception, to modern human history - spanning the rise of the nation state, industrialization, access to mass transportation, the internet and most recently the social media revolution. Given the strategic, adaptive nature of human beings, we can only speculate as to new possible streams of norms, institutions and organizational complexity with subsequent, catastrophic system order shifts and dynamics that might emerge.

Our next research step is to thoroughly empirically verify the entire HDD model with upcoming available WVS data. Once validated, a natural extension is to specifically incorporate agent memory and learning following Quek et al. (2009) and multi-player PD from evolutionary game theory to map the complex transaction networks that emerge over time. Another is to tackle far-from-equilibrium challenges by exploring the full dynamic characteristics of both societal systems and individuals. Key will be identifying behavioral symmetry breaks and formally testing for catastrophic or chaotic responses (Wolf et al. 1985) to economic, cultural, social and political crisis (Abdollahian and Kang 2008), coupling or decoupling. One approach is to endogenize perturbations and shocks across $Y, R S, S E$ and $D$ values and agent space, to map equilibrium stability and system phase transitions. As domain linkages couple and morph, differential patterns of individual behavioral and system response can be identified through multiple local and global solution trajectories. This can allow us to parameterize the entire HDD macromicro model to explicitly test for fold, cusp, swallowtail and higher order catastrophic (Çambel 1993) or chaotic, observed system behavior via Lyapunov exponents (Pecora and Carroll 1990), driven by micro-level interactions.

While only an initial, rough approximation at the truly complex, interdependent and highly nonlinear nature of modernization, our HDD approach provides insights into the interactivity of individual agency and societal outcomes seen through the lens of evolutionary games. While high risk for implementing properly, the knowledge rewards from fusing more detailed and leading theories from economics, cultural studies, sociology and political science via complex adaptive systems methods can be substantial. We hope such work motivates others to extend potential inquiries and insights with even greater theoretical fidelity and empirical resolution. Perhaps techno-social simulations like HDD can assist policy makers and scholar alike, to better understand, anticipate and shape positive social outcomes for all. 
Competing interests

The authors would like to declare that there are no competing interests.

\section{Authors' contributions}

The authors of this paper contributed to the work in the following way: MA, ZY, TC and BY were involved in the conceptualization of HDD. MA and ZY architected the agent based model. ZY instantiated and coded the software implementation in NetLogo. All authors contributed to the manuscript.

\section{Acknowledgments}

The authors would like to acknowledge Hana Oh for her contributions to previous HD system dynamics work, support from the Trans Research Consortium and the anonymous referees for their excellent suggestions.

\section{Author details}

${ }^{1}$ School of Politics and Economics, Claremont Graduate University, 170 E. Tenth St. Claremont, CA 91711, USA. ${ }^{2}$ School of Law, Harvard University, 1563 Massachusetts Ave. Cambridge, MA 02138, USA. ${ }^{3}$ Hatfield School of Government, Portland State University, 1825 SW Broadway Portland, OR 97201, USA.

Received: 22 June 2013 Accepted: 4 September 2013

Published: 08 Nov 2013

\section{References}

Abdollahian M, Kang K: In search of structure: the nonlinear dynamics of power transitions. International Interactions 2008, 34(4):333-357.

Abdollahian M, Coan T, Oh H, Yesilda B: Dynamics of cultural change: the human development perspective. Int Stud Q 2012, 56(4):1-17.

Acemoglu D, Robinson J: Why nations fail: the origins of power, prosperity, and poverty. New York, NY: Crown Business; 2012.

Arthur WB: Positive feedbacks in the economy. Scientific American 1990, 262(2):92-99.

Arthur WB (Ed): Increasing returns and path dependence in the economy. Ann Arbor, MI: University of Michigan Press; 1994.

Axelrod R: The evolution of strategies in the iterated prisoner's Dilemma. In Genetic algorithms and simulated annealing. Edited by Davis L. Los Altos: CA: Morgan Kaufman; 1987:32-41.

Axelrod R: The complexity of cooperation: agent-based models of competition and collaboration. Princeton, NJ: Princeton University Press; 1997a.

Axelrod R: The dissemination of culture: a model with local convergence and global polarization. I Confl Resolut 1997b, 41:203-26.

Barnett T: The pentagon's new map: war and peace in the twenty-first century. New York, NY: J.P. Putnam's Sons; 2004.

Barro R: Economic growth in a cross section of countries. Q J Econ 1991, 106(2):407-444.

Bell D: The coming of postindustrial society. New York, NY: Basic Books; 1973.

Binmore KG: Game theory and the social contract. Cambridge, MA: MIT Press; 1998.

Boix C, Stokes SC: Endogenous democratization. World Politics 2003, 55(4):517-549.

Çambel AB: Applied chaos theory: a paradigm for complexity. San Diego, CA: Academic Press Inc; 1993.

Caselli F, Esquivel G, Lefort F: Reopening the convergence debate: a new look at cross-country growth empirics. J Econ Growth 1996, 1(3):363-389.

Chua A: World on fire. New York, NY: Doubleday; 2003.

Coan T: Participation at the edge of chaos: a study of the nonlinear dynamics of protest politics. PhD thesis. Claremont Graduate University, School of Politics and Economics; 2011.

Darity W: Social judgment theory. Detroit, MI: Macmillan; 2008.

Diamond L: Economic development and democracy reconsidered. In Reexamining Democracy. Edited by Diamond L, Marks G. London, UK: Sage; 1992.

Dixit A, Reiley D, Skeath S: Games of strategies. New York, NY: Norton \& Company; 2009.

Eckstein H, Gurr TR: Patterns of authority: a structural basis for political inquiry. New York, NY: John Wiley \& Sons; 1975. Fehr E, Fischbacher U: Social norms and human cooperation. Trends Cogn Sci 2004, 8:185-190.

Feng Y: Democracy, governance, and economic performance: theory and evidence. Cambridge, MA: The MIT Press; 2003. Fudenberg D, Maskin E: The folk theorem in repeated games with discounting or with incomplete information. Econometrica 1986, 50:533-554.

Griffin E: A first look at communication theory. Boston, MA: McGraw-Hill Higher Education; 2009.

Gurr TR: Why men rebel. Princeton, NJ: Princeton University Press; 1970.

Huntington SP: The clash of civilizations and the remaking of world order. New York, NY: Simon \& Schuster; 1996.

Inglehart R: Modernization and postmodernization: cultural, economic and political change in 43 societies. Princeton, NJ: Princeton University Press; 1997.

Inglehart R, Baker WE: Modernization, cultural change, and the persistence of traditional values. Am Sociol Rev 2000, 65:19-51.

Inglehart R, Welzel C: Modernization, cultural change, and democracy: the human development sequence. New York, NY: Cambridge University Press; 2005.

Kauffman SA: The origins of order: self-organization and selection in evolution. Oxford, UK: Oxford University Press; 1993. Lucas RE Jr: On the mechanics of economic development. Journal of monetary economics 1988, 22(1):3-42.

Mankiw NG, Romer D, Weil DN: A contribution to the empirics of economic growth. Q J Econ 1992, 107(2):407-437.

Maslow A: Motivation and personality. 3rd edition. New York, NY: Harper and Row; 1954.

Maynard-Smith J, Szathmary E: The major transitions in evolution. Oxford, UK: Freeman; 1995. 
McKelvey B: Avoiding complexity catastrophe in coevolutionary pockets: strategies for rugged landscapes. Organizational Science 1999, 10:294-321.

McPherson M, Smith-Lovin L, Cook J: Birds of a feather: homophily in social networks. Annu Rev Sociol 2001, 27:415-44.

Miller JH, Page SE: Complex adaptive systems: an introduction to computational models of social life. Princeton, NJ: Princeton University Press; 2007.

Moyano LG, Sanchez A: Spatial prisoner's dilemma with heterogeneous agents: cooperation, learning and coevolution. Arxiv preprint arXiv 2008:0805-2071.

Norris P, Inglehart R: Sacred and secular: religion and politics worldwide. Cambridge, UK: Cambridge University Press: 2004.

Nowak MA, Sigmund K: Strategy of win-stay, lose-shift that outperforms tit-for-tat in the prisoner's dilemma game. Nature 1993, 364:56-58.

Nowak MA, Sigmund K: Evolution of indirect reciprocity by image scoring. Nature 1998, 1998(393):573-577.

Osterkamp KM: The stability of cooperation in evolutionary games played by finite automata. PhD thesis. Claremont Graduate University, School of Politics and Economics; 1997.

Pecora LM, Carroll TL: Synchronization in chaotic systems. Phys Rev Lett 1990, 64(8):821-824.

Prigogine I, Stengers I: Order out of chaos: man's new dialogue with nature. New York, NY: Bantam Books; 1984.

Quek HY, Tan KC, Abbass HA: Evolutionary game theoretic approach for modeling civil violence. IEEE Trans Evol Comput 2009, 13(4):1-21.

Rodrik D: Globalization, social conflict, and economic growth. The World Economy 1998, 21:143-158.

Romer PM: Increasing returns and long-run growth. The Journal of Political Economy 1986, 94:1002-1037.

Sachs JD: The end of poverty: economic possibilities for our time. New York, NY: Penguin Books; 2005.

Sala-i-Martin XX: Regional cohesion: evidence and theories of regional growth and convergence. Eur Econ Rev 1996, 40(6):1325-1352.

Santos FC, Pacheco JM, Lenaerts T: Evolutionary dynamics of social dilemmas in structured heterogeneous populations. Proc Natl Acad Sci USA 2006, 103(9):3490-3494.

Schelling T: Dynamic models of segregation. The Journal of Mathematical Sociology 1971, 1:143-186.

Siero FW, Doosje BJ: Attitude change following persuasive communication: integrating social judgment theory and the elaboration likelihood model. European journal of social psychology 1993, 23(5):541-554.

Sigmund K: Games of life: Oxford. UK: Oxford University Press; 1993.

Sigmund K, Nowak MA: Evolutionary game theory. Current Biology 1999, 9(14):503-505.

Snijders TA, Steglich CE, Schweinberger M: Modeling the co-evolution of networks and behavior. Longitudinal Models in the Behavioral and Related Sciences; 2007:41-71.

Solow R: A contribution to the theory of economic growth. The Quarterly Journal of Economics 1956, 70(1):65-94.

Swan T: Economic growth and capital accumulation. Economic Record 1956, 32(2):334-361.

Thom R: Structural stability and morphogenesis. Bull Math Biol 1977, 39(5):629-632.

Vespignani A: Predicting the behavior of techno-social systems. Science 2009, 325:425-428.

Welzel C, Inglehart R, Klingemann HD: The theory of human development: a cross-cultural development. European Journal of Political Science 2003, 42:341-380.

Wilensky U: NetLogo. Northwestern University, Center for Connected Learning and Computer-Based Modeling; 1999. http://ccl.northwestern.edu/netlogo/.

Wolf A, Swift JB, Swinney HL, Vastano JA: Determining lyapunov exponents from a time series. Physica D: Nonlinear Phenomena 1985, 16(3):285-317.

Zheleva E, Sharara H, Getoor L: Co-evolution of social and affiliation networks, Proceedings of the 15th ACM SIGKDD international conference on knowledge discovery and data mining. New York: NY: ACM; 2009.

10.1186/2194-3206-1-18

Cite this article as: Abdollahian et al:: Human development dynamics: an agent based simulation of macro social systems and individual heterogeneous evolutionary games. Complex Adaptive Systems Modeling 2013, 1:18

\section{Submit your manuscript to a SpringerOpen ${ }^{\circ}$ journal and benefit from:}

- Convenient online submission

Rigorous peer review

- Immediate publication on acceptance

- Open access: articles freely available online

- High visibility within the field

Retaining the copyright to your article

Submit your next manuscript at $\boldsymbol{~ s p r i n g e r o p e n . c o m ~}$ 